\title{
Assessment of different NEoplasias in the adneXa model for differentiation of benign and malignant adnexal masses in Korean women
}

\author{
Gina Nam, MD¹, Sa Ra Lee, MD, PhD², Kyungah Jeong, MD, PhD³ , Sung Hoon Kim, MD, PhD², \\ Hye-Sung Moon, MD, PhD², Hee Dong Chae, MD, $\mathrm{PhD}^{2}$ \\ Department of Obstetrics and Gynecology, ${ }^{1}$ Chung-Ang University Hospital, Chung-Ang University College of Medicine, ${ }^{2}$ Asan Medical Center, \\ University of Ulsan College of Medicine, ${ }^{3}$ Ewha Womans University School of Medicine, Seoul, Korea
}

\section{Objective}

Ultrasonographic differential diagnosis of ovarian tumors is important for appropriate management. We conducted study to compare the performance of the Assessment of Different NEoplasias in the adneXa (ADNEX) model with a subjective assessment (SA) in differentiating between benign and malignant adnexal masses in Korean women.

\section{Methods}

A total of 353 patients who underwent adnexal surgery with abnormal pelvic ultrasonographic findings from August 2016 to August 2017 were included in study. The presumptive diagnosis of adnexal malignancy was determined by both SA and the ADNEX model to be $>10 \%$ calculated risk of malignancy. The area under the curve (AUC) comparison between the SA and ADNEX models was performed using DeLong's method.

\section{Results}

340 patients with benign tumors and 13 with malignant adnexal tumors among $292(82.72 \%)$ premenopausal and 61 (17.28\%) postmenopausal women were included. The AUCs of SA and the ADNEX model for discrimination between benign and malignant tumors were 0.79 and 0.92 , respectively $(P=0.10)$. The sensitivity and specificity of SA and the ADNEX model were $83.5 \%$ and $97.0 \%$, and $90.0 \%$ and $82.0 \%$, respectively. Comparison of the ADNEX model regarding menopausal status revealed that the predictability was not different. The AUCs of SA and the ADNEX model in premenopausal women were 0.74 and 0.89 , respectively $(P=0.12)$. The AUCs of SA and the ADNEX model in postmenopausal women were 0.86 and 0.94 , respectively $(P=0.60)$.

\section{Conclusion}

The ADNEX model offers excellent discrimination between benign and malignant ovarian tumors with similar sensitivity and specificity to SA in both premenopausal and postmenopausal Korean women.

Keywords: Ovarian neoplasms; Fertility preservation; Prediction model; Ultrasonography

Received: 2021.01.02. Revised: 2021.01.29. Accepted: 2021.02.08.

Corresponding author: Sa Ra Lee, MD, PhD

Department of Obstetrics and Gynecology, Asan Medical Center, University of Ulsan College of Medicine, 88 Olympic-ro 43-gil, Songpagu, Seoul 05505, Korea

E-mail: leesr@amc.seoul.kr

https://orcid.org/0000-0002-7890-8348

Kyungah Jeong has been an Editorial Board of Obstetrics \& Gynecology Science; however, she was not involved in the peer reviewer selection, evaluation, or decision process of this article. Otherwise, no other potential conflicts of interest relevant to this article was reported. 


\title{
Obstetrics \& Gynecology Science
}

\author{
Vol. 64, No. 3, 2021
}

\section{Introduction}

Ultrasonographic differential diagnosis of benign or malignant ovarian tumors is important for appropriate management, including diagnostic work-up, referral to tertiary centers, and surgical plans. Benign ovarian tumors can be treated with ovarian cystectomy for the preservation of ovarian function; this surgical option is especially important for women who want to preserve fertility for future pregnancy. Early diagnosis and surgical treatment are necessary to achieve a good prognosis for ovarian cancer, which is known to have the worst prognosis among gynecological cancers. Among gynecologic cancers, ovarian cancer is the second most common, and the incidence of ovarian cancer between 1999 and 2015 in Korea has been gradually increasing [1]. The five-year survival rate of ovarian cancer is approximately $40 \%$, and the disease accounts for approximately half of all deaths related to gynecological cancer. The most important factor for survival is the stage at diagnosis [2]. However, sonographic differential diagnosis of ovarian tumors is still difficult, even in recent years, with marked improvements in ultrasonography technology. Obtaining the right sonographic view and interpreting the sonographic images are somewhat subjective. The accuracy of sonographic diagnosis of malignant adnexal tumors is thus highly dependent on the experience of the person performing sonography.

Therefore, attempts have been made to determine an evidence-based approach for the diagnosis and management of adnexal masses, and the International Ovarian Tumor Analysis (IOTA) group studied the classification of ovarian masses [3]. Simple ultrasound-based rules (simple rules), the risk of malignancy index (RMI), and the risk of ovarian malignancy algorithm (ROMA) have been reported [4,5]. Recently, the IOTA group issued a polytomous risk prediction model for the classification of adnexal masses, the Assessment of Different NEoplasias in the adneXa (ADNEX) model [2]. This model considers three clinical and six ultrasound predictors: age, serum CA-125 level, type of center (referral center for oncology or not), maximum diameter of lesion, proportion of solid tissue, more than 10 cyst locules, number of papillary projections, acoustic shadows, and ascites. The ADNEX model is the first risk model that differentiates between benign and four types of malignant ovarian tumors and predicts the classification of adnexal masses into five tumor categories: benign, borderline, stage I ovarian cancer, stage II-IV ovarian cancer, and secondary ovarian metastasis $[2,6]$. The prediction, which focuses not only on the presence of malignancy but also the degree of malignancy, can inform decisions regarding the type of surgery. Benign ovarian masses can be managed conservatively or with surgical intervention $[7,8]$. Borderline ovarian tumors are associated with remarkable survival, almost $100 \%$ within 10 years [9]. Additionally, less aggressive surgical treatment should be considered than that for invasive tumors, and fertility-sparing procedures are especially important for reproductive age women $[10,11]$. Among malignant ovarian tumors, early stage ovarian cancer may be managed less invasively than advanced-stage disease. For example, patients with early stage ovarian cancer may undergo laparoscopic exploration instead of laparotomy $[12,13]$. Women with ovarian metastasis need to be treated depending on the type of the primary origin of the tumor, and they require specific diagnostic and therapeutic management $[14,15]$.

In terms of ovarian tumors, there can be racial differences, and studies among European women have validated the ADNEX model; however, as far as we know, there have been no previous studies on Asian women in general and Korean women in particular. Therefore, the aim of this study was to compare the performance of the ADNEX model with the subjective assessment (SA) of gynecologists in differentiating between benign and malignant adnexal masses in Korean women.

\section{Materials and methods}

This retrospective diagnostic accuracy study was conducted with prospectively collected data from patients with adnexal pathology who underwent transvaginal sonography or transrectal sonography from August 2016 to August 2017 and who underwent adnexal surgery by three gynecologic surgeons (S.R.L., K.J., and H.S.M) with confirmed pathology from Ewha Womans University Mokdong Hospital. The presence of a malignancy was determined by both SA and the ADNEX model (malignancy risk $>10 \%$ ). Ultrasound examinations were performed using a WS80A instrument (Samsung Medison Company, Seoul, Korea) with a VR5-9 probe. All preoperative pelvic sonographic images were reviewed by a single gynecologic surgeon (S.R.L.) with a personal history of more than 16 years of gynecologic sonographic examina- 


\section{Obstetrics \& Gynecology Science}

Gina Nam, et al. Ultrasonographic differential diagnosis model of adnexal mass

tion in a tertiary center. Surgical pathologic diagnosis was the clinical reference standard. A total of 390 consecutive patients who underwent preoperative ultrasound examination for adnexal masses and had surgically proven pathologic results were initially included in the sample. The exclusion criteria were age below 13 years and malignancy from other (i.e., nongynecologic) origins, such as metastatic or peritoneal cancer. A total of 353 consecutive cases, 292 (82.72\%) premenopausal and 61 (17.28\%) postmenopausal women, were included in this analysis. The pathological distribution of tumors was 340 (96.3\%) benign and 13 (3.7\%) malignant tumors. We calculated the ADNEX model using the formula provided on the ADNEX webpage (http://www.iotagroup. org/adnexmodel/) and used a cutoff of $10 \%$ to estimate the risk of malignancy. We compared the under the curve (AUC) between the SA and ADNEX models using DeLong's test. All computations were performed with $\mathrm{R}$, a language and environment for statistical computation ( $R$ Foundation for Statistical Computing, Vienna, Austria) [16]. All tests were considered significant at the 0.05 level. This study protocol was approved by the Institutional Review Board (approval no. EUMC 2021-03-009).

\section{Results}

Overall, the mean age and standard deviation (SD) of the patients was $38.5 \pm 14.0$ years. The mean tumor maximal diameter was $66.0 \pm 38.0 \mathrm{~mm}$, and the mean CA-125 level was $53.5 \pm 118.2 \mathrm{IU} / \mathrm{mL}$. The mean age and SD of patients for whom the risk of malignancy was $>10 \%$ in the ADNEX model was $40.6 \pm 16.8$ years. Among these patients, the mean tumor maximal diameter was $91.8 \pm 55.5 \mathrm{~mm}$ and the mean
CA-125 level was $119.2 \pm 239.0 \mathrm{IU} / \mathrm{mL}$. The mean age and SD of patients for whom the risk of malignancy was $\leq 10 \%$ in the ADNEX model was $35.6 \pm 12.0$ years, the mean tumor maximal diameter was $59.0 \pm 28.6 \mathrm{~mm}$, and the mean CA125 level was $36.3 \pm 44.8 \mathrm{IU} / \mathrm{mL}$ (Table 1).

The sensitivity and specificity AUC comparison with DeLong's method was not significant $(P=0.10)$. This means that the ADNEX model ( $A U C=0.92$ ) is comparable to the SA model $(A \cup C=0.79)$. In terms of sensitivity and specificity for

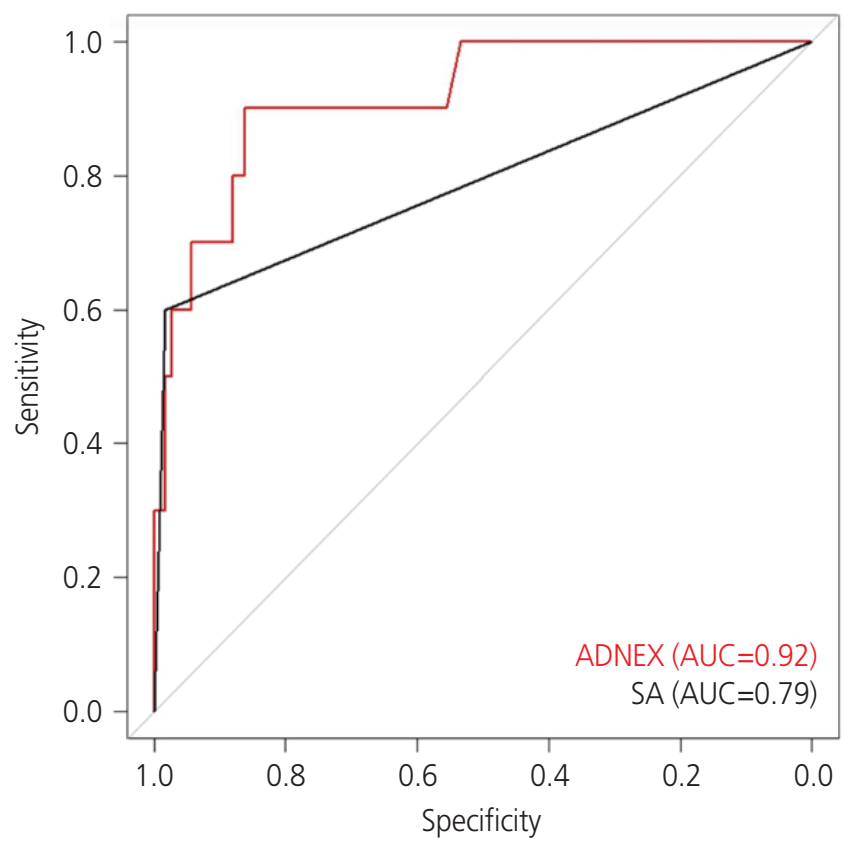

Fig. 1. The area under the receiver operating curve (AUC) comparison between the Assessment of Different NEoplasias in the adneXa (ADNEX) model (red line) and a subjective assessment (SA) (black line) in differentiation between benign and malignant adnexal masses in Korean women.

Table 1. Descriptive information about the patient characteristics and ultrasound features according to malignant risk of ADNEX model

\begin{tabular}{|c|c|c|c|}
\hline & \multicolumn{3}{|c|}{ Malignancy risk of ADNEX model } \\
\hline & $>10 \%$ & $\leq 10 \%$ & Total \\
\hline Age (yr) & $40.6 \pm 16.8$ & $35.6 \pm 12.0$ & $38.5 \pm 14.0$ \\
\hline CA-125 (IU/mL) & $119.2 \pm 239.0$ & $36.3 \pm 44.8$ & $53.5 \pm 118.2$ \\
\hline The maximal diameter of tumor (mm) & $91.8 \pm 55.5$ & $59.0 \pm 28.6$ & $66.0 \pm 38.0$ \\
\hline Largest solid part (mm) & $30.5 \pm 20.7$ & $8.1 \pm 3.8$ & $24.0 \pm 20.5$ \\
\hline Number of papillary projections & $2.5 \pm 0.7$ & 0 & $2.5 \pm 0.7$ \\
\hline
\end{tabular}

Data are presented as mean \pm standard deviation.

ADNEX, Assessment of Different NEoplasias in the adnexa; CA-125, cancer antigen 125. 


\section{Obstetrics \& Gynecology Science}

Vol. 64, No. 3, 2021

the group in which the ADNEX model malignancy risk was $>10 \%$, the sensitivity and specificity of SA and the ADNEX model were $83.5 \%$ and $97.0 \%$ and $90.0 \%$ and $82.0 \%$, respectively (Fig. 1). Comparison of the ADNEX model with respect to menopausal status revealed that the predictive validity was not different. For premenopausal women, the AUC comparison was not significant $(P=0.12)$, and the sensitivity and specificity for the group in which the ADNEX model malignancy risk was $>10 \%$ were $83 \%$ and $83 \%$, respectively. For postmenopausal women, the AUC comparison was not significant $(P=0.60)$, and the sensitivity and specificity for the group in which the ADNEX model malignancy risk was $>10 \%$ were $100 \%$ and $76 \%$, respectively (Fig. 2).

\section{Discussion}

This study showed that the ADNEX model results in an excellent differentiation between benign and malignant adnexal masses, with an AUC of 0.92 , with a similar sensitivity and specificity as SA. Studies on the external validation of the ADNEX model have reported a similar good performance [17-21]. A prospective multicenter study was performed in 24 ultrasound centers in 10 countries that included many patients $(n=5,909)[2]$. The authors reported that the AUC of the ADNEX model for classic discrimination between benign and malignant tumors was 0.94 (95\% confidence interval [Cl], 0.93-0.95) [2]. A Dutch study including 326 patients (128 premenopausal and 198 postmenopausal women) concluded that the ADNEX model, with an AUC of 0.93 (95\% $\mathrm{Cl}, 0.89-0.95)$, had good overall performance in comparison with other ultrasonographic scoring methods, simple rules, logistic regression model 2, and RMI [18].

A study evaluating the ADNEX model in 93 French postmenopausal women concluded that the ADNEX model can increase the utilization of pelvic ultrasound and distinguish malignant cysts from ovarian tumors [19]. Recent articles concluded that the ADNEX model can be used to improve surgical strategy $[2,20]$. Accurate prediction of ovarian tumors between four types of ovarian malignancy-borderline, stage I, stage II-IV, and metastasis from malignancies of other organs-can improve triage and management decisions. Therefore, the ADNEX model can help in planning the most appropriate surgical approach, minimally invasive surgery or explo-laparotomy.

Few studies have reported a lack of usefulness of the ADNEX model. One study suggested that the ADNEX model has limitations in predicting ovarian tumor types [17]. The Dutch study also mentioned that SA by an expert, a single ultrasonographer with more than 10 years of experience in
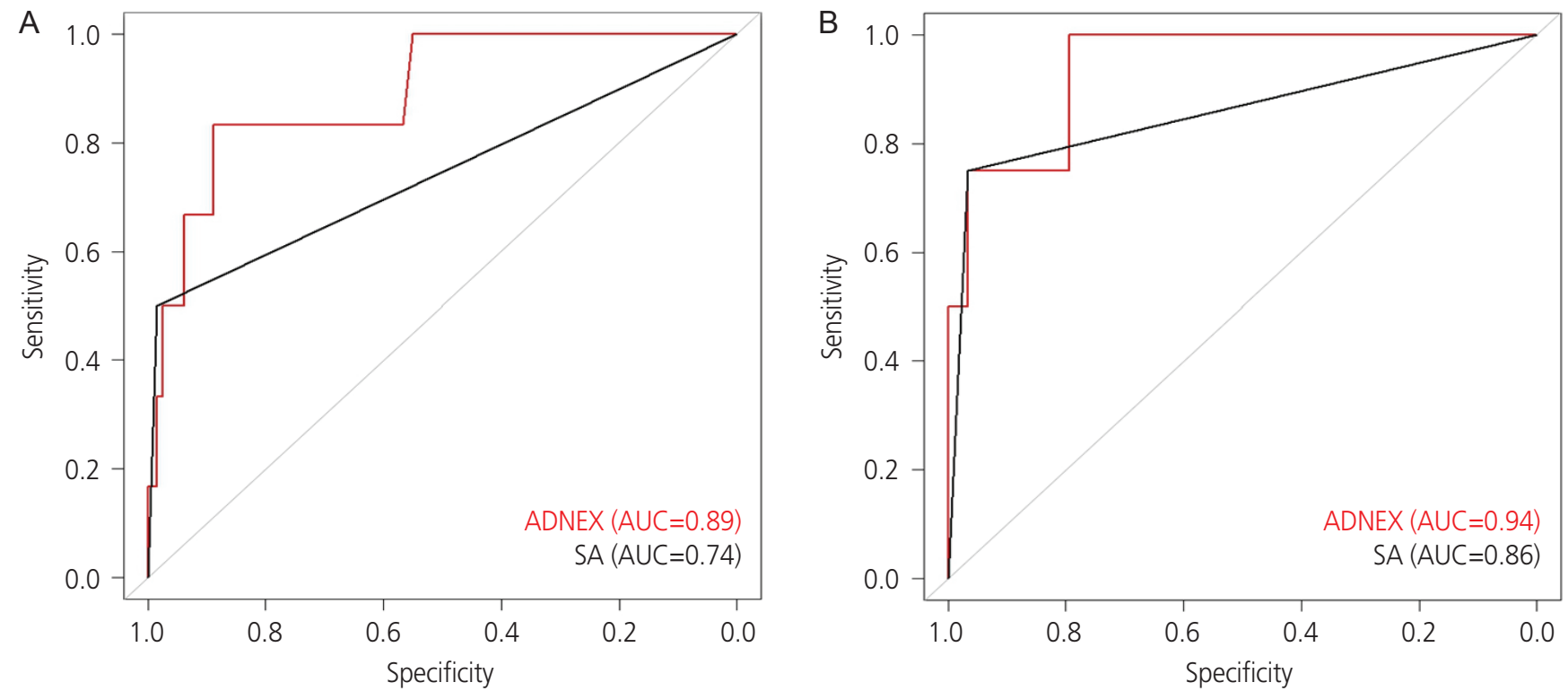

Fig. 2. The area under the receiver operating curve (AUC) comparison between the Assessment of Different NEoplasias in the adneXa (ADNEX) model (red line) and a subjective assessment (SA) (black line) in differentiation between benign and malignant adnexal masses in Korean (A) premenopausal women and (B) postmenopausal women. 


\section{Obstetrics \& Gynecology Science}

Gina Nam, et al. Ultrasonographic differential diagnosis model of adnexal mass

gynecological ultrasonography (level 3 examiner), remains superior to the ADNEX model, and the advantage of the ADNEX model as a polytomous model needs to be proven in future studies [18]. A prospective study including 610 patients from 3 countries concluded that the ADNEX model can be useful even in the absence of CA-125 levels [20]. The authors reported that the diagnostic performance of ADNEX in differentiating between benign and malignant masses was $0.937(95 \% \mathrm{Cl}, 0.915-0.954)$ when CA-125 was included and $0.925(95 \% \mathrm{Cl}, 0.902-0.943)$ when CA-125 was excluded [20]. This can be a useful advantage of the ADNEX model considering that the CA-125 level can only be obtained several hours after a blood test, a relatively invasive procedure. Conversely, we can predict the malignancy of adnexal masses as soon as the ADNEX model is applied, without delay or invasive procedures.

This study has several strengths. First, to the best of our knowledge, this is the first study to compare SA with the ADNEX model among Asian women. Second, the relatively short study period of 13 months precludes intrapersonal variation in sonographic experiences. Third, the number of patients was relatively large among studies on the ADNEX model, except for the original diagnostic multicenter study [2].

However, this study also had limitations that should be considered. First, most patients were premenopausal women, and the proportion of postmenopausal women, who have a higher prevalence of malignant adnexal tumors, was less than $20 \%$. Second, the proportion of malignant ovarian tumors was low $(3.7 \%)$, considering that this study was performed by one oncologic surgeon and two reproductive endocrinologic surgeons in a tertiary gynecologic oncology center. The general malignancy rate in a previous report was $27 \%$ [2]. Moreover, this study was conducted in a thirdlevel center, while the previous study was performed in both second- and third-level hospitals. However, most gynecologic oncology centers in South Korea perform gynecologic surgeries for both benign and malignant conditions. Therefore, the low proportion of malignancy can be an advantage when generalizing this study's results to private clinics, which are more prevalent than tertiary centers. One of the purposes of developing the ADNEX model was to help make decisions with an evidence-based adjunct, even by the hands of nonexpert ultrasound examiners. This can also be useful for less developed countries with few experts in ultrasonography.
However, each country's referral system is different. Therefore, the variable "type of center," defined as a tertiary referral center with a specific gynecology oncology unit, must be interpreted differently in each medical system.

The proportion of borderline and malignant tumors in our study was comparable to that in a study of postmenopausal women, with $3.2 \%$ malignancies and $1.1 \%$ borderline malignancies [19]. Third, this study only compared differentiation between benign and malignant adnexal masses, and we did not include the differentiation among borderline malignancies, stage I ovarian cancer, stage II-IV ovarian cancer, or metastatic cancers. Although the polytomous approach to adnexal tumor diagnosis is a novel component of the ADNEX model, previous reports raised questions about the clinical utility of discriminating stages I and II-IV. The final diagnosis and postoperative treatment plan should be based on the pathologic diagnosis and proven prognostic factors. Furthermore, a study including 327 patients in 2 countries also reported only moderate accuracy in the prediction of ovarian tumor types (sensitivity, 64.2\%; specificity, $74.0 \%$ ) in addition to a very high accuracy in differentiating malignant and benign adnexal tumors (sensitivity $79.9 \%$, specificity $81.3 \%$ ) [21].

We must keep in mind that prior to the application of the ADNEX model, physicians should be well trained in basic gynecologic sonographic findings. Only under this condition can nonexpert ultrasound examiners obtain an accurate prediction of adnexal tumors using the ADNEX model. The reason for this is that six out of the nine components of the ADNEX model are sonographic predictors that are relatively subjective. In conclusion, the ADNEX model offers excellent discrimination of benign and malignant ovarian tumors with similar sensitivity and specificity to SA by experienced gynecologists. Gynecologists can be assisted to conduct management plan decisions with the use of the ADNEX model in both premenopausal and postmenopausal women.

\section{Conflict of interest}

No potential conflict of interest relevant to this article was reported. 


\section{Obstetrics \& Gynecology Science}

Vol. 64, No. 3, 2021

\section{Ethical approval}

This study protocol was approved by the Institutional Review Board (approval no. EUMC 2021-03-009). The study was performed in accordance with the principles of the Declaration of Helsinki.

\section{Patient consent}

Written informed consent and the use of images from patients are not required for the publication.

\section{Funding information}

The work was funded by Samsung Medison Company (Seoul, Korea) and the Institute for National IT Industry Promotion Agency (NIPA) grant funded by the Korea government (MSIT) (No. A0602-19-1032, Intelligent surgical guide system \& service from surgery video data analytics).

\section{Acknowledgement}

This article was partly presented at the 20th KSUOG annual conference (Busan, Korea) on November 4, 2017.

\section{References}

1. Lim MC, Won YJ, Ko MJ, Kim M, Shim SH, Suh DH, et al. Incidence of cervical, endometrial, and ovarian cancer in Korea during 1999-2015. J Gynecol Oncol 2019;30:e38.

2. Van Calster B, Van Hoorde K, Valentin L, Testa AC, Fischerova $D$, Van Holsbeke $C$, et al. Evaluating the risk of ovarian cancer before surgery using the ADNEX model to differentiate between benign, borderline, early and advanced stage invasive, and secondary metastatic tumours: prospective multicenter diagnostic study. BMJ 2014;349:95920.

3. Kaijser J. Towards an evidence-based approach for diagnosis and management of adnexal masses: findings of the International Ovarian Tumour Analysis (IOTA) stud- ies. Facts Views Vis Obgyn 2015;7:42-59.

4. Jacobs I, Oram D, Fairbanks J, Turner J, Frost C, Grudzinskas JG. A risk of malignancy index incorporating CA 125, ultrasound and menopausal status for the accurate preoperative diagnosis of ovarian cancer. $\mathrm{Br} J$ Obstet Gynaecol 1990;97:922-9.

5. Moore RG, McMeekin DS, Brown AK, DiSilvestro P, Miller MC, Allard WJ, et al. A novel multiple marker bioassay utilizing HE4 and CA125 for the prediction of ovarian cancer in patients with a pelvic mass. Gynecol Oncol 2009;112;40-6.

6. Van Calster B, Van Hoorde K, Froyman W, Kaijser J, Wynants L, Landolfo C, et al. Practical guidance for applying the ADNEX model from the IOTA group to discriminate between different subtypes of adnexal tumors. Facts Views Vis Obgyn 2015;7:32-41.

7. American College of Obstetricians and Gynecologists' Committee on Practice Bulletins-Gynecology. Practice Bulletin No. 174: evaluation and management of adnexal masses. Obstet Gynecol 2016;128:e210-26.

8. Carley ME, Klingele CJ, Gebhart JB, Webb MJ, Wilson TO. Laparoscopy versus laparotomy in the management of benign unilateral adnexal masses. J Am Assoc Gynecol Laparosc 2002;9:321-6.

9. Tropé CG, Kaern J, Davidson B. Borderline ovarian tumours. Best Pract Res Clin Obstet Gynaecol 2012;26:325-36.

10. Tinelli R, Tinelli A, Tinelli FG, Cicinelli E, Malvasi A. Conservative surgery for borderline ovarian tumors: a review. Gynecol Oncol 2006;100:185-91.

11. Daraï E, Fauvet R, Uzan C, Gouy S, Duvillard P, Morice P. Fertility and borderline ovarian tumor: a systematic review of conservative management, risk of recurrence and alternative options. Hum Reprod Update 2013;19:151-66.

12. Trimbos JB, Vergote I, Bolis G, Vermorken JB, Mangioni C, Madronal C, et al. Impact of adjuvant chemotherapy and surgical staging in early-stage ovarian carcinoma: European Organisation for Research and Treatment of Cancer-Adjuvant ChemoTherapy in Ovarian Neoplasm trial. J Natl Cancer Inst 2003;95:113-25.

13. Zhang Y, Fan S, Xiang Y, Duan H, Sun L. Comparison of the prognosis and recurrence of apparent early-stage ovarian tumors treated with laparoscopy and laparotomy: a meta-analysis of clinical studies. BMC Cancer 


\section{Obstetrics \& Gynecology Science}

Gina Nam, et al. Ultrasonographic differential diagnosis model of adnexal mass

2015;15:597.

14. Lee SJ, Bae JH, Lee AW, Tong SY, Park YG, Park JS. Clinical characteristics of metastatic tumors to the ovaries. J Korean Med Sci 2009;24:114-9.

15. Hennessy BT, Coleman RL, Markman M. Ovarian cancer. Lancet 2009;374:1371-82.

16. R Core Team (2020). R: A language and environment for statistical computing [Internet]. Vienna: R Foundation for Statistical Computing; c2020 [cited 2020 Sep 11]. Available from: https://www.R-project.org/.

17. Araujo KG, Jales RM, Pereira PN, Yoshida A, de Angelo Andrade L, Sarian LO, et al. Performance of the IOTA ADNEX model in preoperative discrimination of adnexal masses in a gynecological oncology center. Ultrasound Obstet Gynecol 2017;49:778-83.

18. Meys EMJ, Jeelof LS, Achten NMJ, Slangen BFM, Lambrechts $S$, Kruitwagen RFPM, et al. Estimating risk of malignancy in adnexal masses: external validation of the ADNEX model and comparison with other frequently used ultrasound methods. Ultrasound Obstet Gynecol 2017;49:784-92.

19. Nohuz E, De Simone L, Chêne G. Reliability of IOTA score and ADNEX model in the screening of ovarian malignancy in postmenopausal women. J Gynecol Obstet Hum Reprod 2019;48:103-7.

20. Sayasneh A, Ferrara L, De Cock B, Saso S, Al-Memar M, Johnson $S$, et al. Evaluating the risk of ovarian cancer before surgery using the ADNEX model: a multicenter external validation study. Br J Cancer 2016;115:542-8.

21. Szubert S, Wojtowicz A, Moszynski R, Zywica P, Dyczkowski K, Stachowiak A, et al. External validation of IOTA ADNEX model performed by two independent gynecologic centers. Gynecol Oncol 2016;142:490-5. 